\title{
Beiträge zur Kenntniss der Bestandtheile des Emmenthaler Käses.
}

Von

E. Winterstein 1) und J. Thöny.

(Aus dem agriculturchemischen Laboratorium des Polytechnikums in Zürich.)

(Der Redaction zugegangen am 3. Juli 1902.)

Aus dem hiesigen Laboratorium ist eine Reihe von Untersuchungen ${ }^{2}$ ) hervorgegangen, welche sich mit den beim Reifen harter Fettkäse abspielenden Vorgängen befassten. Durch diese Arbeiten wurden die Stoffumwandlungen, welche die frische Käsemasse während ihrer Reifung erleidet, theilweise aufgeklärt. Es ergab sich, dass der eine Hauptbestandtheil des Emmenthaler Käses, nämlich das Fett, beim Reifen nur wenig verändert wird, während dagegen die Eiweisssubstanz, das Paracasein, ${ }^{3}$ ) welches den allergrössten Theil der stickstoffhaltigen Substanz der Käsemasse ausmacht, eine tiefgreifende Spaltung unter Bildung von Ammoniak, Aminosäuren und alkohollöslichen Eiweisssubstanzen (Caseoglutin) ${ }^{4}$ ) erleidet. Die Veränderung, welche das Paracasein dabei erfährt, ist bei längerer Dauer des Reifungsprocesses in quantitativer ${ }^{5}$ ) Hin-

1) Berichterstatter: E. Winterstein.

2) Untersuchungen über die Zusammensetzung und den Reifungsprocess des Emmenthaler Käses. U. Weidmann, Landwirthschaftl. Jahrbücher, 1882 , S. 587.

Untersuchungen über den Emmenthaler Käse und über einige andere schweizerische Käsesorten. F. Benecke und E. Schulze, ibid., 1887, S. 317.

Ueber einige Bestandtheile des Emmenthaler Käses. B. Röse und E. Schulze, Landwirthschaftl. Versuchsstationen, 1884, S. 115.

3) Als Paracasein bezeichnet E. Schulze die aus der Milch mit Hülfe von Labferment abgeschiedene Eiweisssubstanz.

4) Siehe die Arbeit von Weidmann. S. 594 .

5) Siehe die Arbeit von Weidmann. S. 597. 
sicht eine recht beträchtliche. Unter den bei dieser Spaltung sich bildenden Stoffen konnten Leucin und Tyrosin nachgewiesen und das Vorhandensein von Phenylalanin wahrscheinlich gemacht werden. Dass diese Aminosäuren die einzigen bei dem Zerfall des Paracaseins auftretenden Spaltungsprodukte nicht sein konnten, wurde schon in einer der ersten der oben citirten Arbeiten ${ }^{1}$ ) auf Grund quantitativer Untersuchungen ausgesprochen. Mit Rücksicht auf die in der neuen Zeit gewonnenen Ergebnisse über die hydrolytischen Spaltungsprodukte der Proteinsubstanzen war an der Richtigkeit dieser Vermuthung kaum zu zweifeln. Erstens war anzunehmen, dass aus dem Paracasein neben Aminosäuren auch die inzwischen unter den Spaltungsprodukten der Eiweissstoffe aufgefundenen Basen Arginin, Lysin und Histidin sich bilden; zweitens aber durfte man erwarten, dass bei der während der Käsereifung lange andauernden Einwirkung von Enzymen und von Mikroorganismen die primären krystallinischen Spaltungsprodukte des Paracaseins eine weitere Umwandlung erleiden. Es war von Interesse, diese beiden Fragen zum Gegenstand einer experimentellen Untersuchung zu machen.

Schon vor einigen Jahren hat der Eine von uns, E. Winterstein, im Einverständniss mit Herrn Professor E. Schulze mit einigen Schülern Versuche zur Lösung dieser Frage angestellt. Durch die im Verein mit den Herren A. Rosam und G. Köstler ausgeführten Versuche konnte constatirt werden, dass neben Aminosäuren basische Zersetzungsprodukte in wechselnder Quantität in den Käsesorten verschiedener Provenienz und von verschiedenem Alter sich nachweisen lassen; es gelang auch, das Vorhandensein von Histidin zu constatiren, der Nachweis von Arginin und Lysin war damals nicht gelungen. Es wurde nämlich bei diesen Versuchen die Erfahrung gemacht, dass wegen der kleinen Ausbeute an basischen Eiweisszersetzungsprodukten nur bei Verarbeitung einer relativ

1) Untersuchungen über die Zusammensetzung und den Reifungsprocess des Emmenthaler Käses. U. Weidmann, Landwirthschaftl. Jahrbücher, 1882 , S. 598 . 
grossen Quantität Käse eine zur Untersuchung genügende Quantität jener Stoffe gewonnen werden konnte. Unter diesen Umständen musste es unsere nächste Aufgabe sein, eine grössere Quantität Käse in Arbeit zu nehmen. Wir verwendeten circa $6 \frac{1}{2}$ Kilo eines fehlerhaften sogenannten geblähten und randhohlen Emmenthaler Käses.

\section{Beschreibung des bei der Verarbeitung des Käses eingehaltenen Verfahrens.}

Der von der äusseren Rinde befreite Käse wurde fein geraspelt, an der Luft ausgetrocknet und im 'Thörner'schen Extractionsapparat mit Aether möglichst entfettet, die nahezu fettfreie Masse wurde in dünne Schichten ausgebreitet. Nach dem Verdunsten des Aethers hinterblieb eine weisse, leicht zerreibliche Masse; dieselbe wurde mit der $4-5$ fachen Menge 80\% igem Weingeist mehrere Stunden gekocht; durch rasches Abkühlen erzielt man, dass das Ungelöste sich von der alkoholischen gelblichen Lösung rasch sondert; diese Lösung wird abgegossen, der Rüchstand vorsichti; abgepresst und darauf noch 4-5 mal, wie angegeben, behandelt. Auf diese Weise werden die alkohollöslichen Eiweissstoffe, nebst den Aminosäuren nahezu vollständig in Lösung gebracht. Die nach Extraction mit Alkohol verbliebene Masse wurde mit Wasser in der Wärme behandelt, gut abgepresst und die wässerige Lösung eingedunstet und ebenso weiter behandelt, wie das vom Alkohol befreite Extract. (Siehe weiter unten.) Die vereinigten Extracte wurden durch Destillation nahezu vom Alkohol befreit und der hellbraune Destillationsrückstand unter ständigem Umrühren in viel Wasser gegossen, wobei ein flockiger, sich rasch zu Boden setzender Niederschlag auftrat. Diese Substanz stimmt in ihrem Verhalten mit Caseoglutin überein, die davon durch Filtration getrennte Flüssigkeit wurde auf dem Wasserbade bis zum Beginn der Krystallisation eingedunstet, wobei eine amorphe, leimartige, fest an den Wandungen haftende Substanz sich ausschied, ${ }^{1}$ ) von welcher die Flüssigkeit nach dem Erkalten abgegossen werden konnte.

1) Diese Substanz gab die für Eiweiss charakteristischen Reactionen. 
Aus der dickflüssigen Lösung, welche nur noch kleine Mengen von Eiweisssubstanzen enthielt, wurden durch Krystallisation fünf Fractionen von Aminosäuren hergestellt. Die von der letzten Krystallisation getrennte Mutterlauge wurde mit Gerbsäure ausgefällt. Aus dem Gerbsäureniederschlag konnten durch Zersetzen mit Baryt Peptone isolirt werden. Die vom Tanninniederschlag abfiltrirte Lösung wird nun tropfenweise mit Bleiessig versetzt, bis eine abfiltrirte Probe mit diesem Reagens nur noch eine schwache Trübung erzeugt; der entstandene Niederschlag wurde auf ein Filter gebracht, mit Wasser gut ausgewaschen, sodann nochmals mit Wasser gut zerrieben und nochmals durch Filtration von der Flüssigkeit getrennt. Die Filtrate und Waschwässer werden mit Schwefelsäure vom Blei befreit, die vom Bleisulfat getrennte Lösung wurde auf dem Wasserbade bei niederer Temperatur concentrirt und sodann mit einer 50\% igen Lösung von Phosphorwolframsäure ausgefällt. Auf Zusatz von Phosphorwolframsäure erfolgt anfänglich eine starke, sich rasch absetzende krystallinische Fällung, bei weiterem Zusatz des genannten Reagenses tritt ein äusserst feiner Niederschlag auf, welcher in der Flüssigkeit tagelang suspendirt bleibt. Durch Absaugen konnte der fein vertheilte Niederschlag nicht von der Flüssigkeit getrennt werden. Er wurde daher auf mehrere Filter gebracht, mit einem Gemisch von $3 \%$ iger Schwefelsäure und Phosphorwolframsäure ausgewaschen, sodann zwischen Fliesspapier getrocknet. Die farblose, seidenglänzende krystallinische Phosphorwolframatverbindung wurde in bekannter Weise mit alkalifreiem Barythydrat zersetzt und die erhaltene Basenlösung durch Einleiten von Luft vom Ammoniak und gleichzeitig vom überschüssigen Baryt befreit. (Die farblose Basenlösung gab mit Nessler'schem Reagens eine dicke, schwach gelb gefärbte Fällung.) Diese alkalisch reagirende Flüssigkeit musste das Arginin, das Histidin und das Lysin enthalten. Behufs Trennung der basischen Produkte benutzten wir das ältere Verfahren von A. Koss el. ${ }^{1}$ ) Die mit Kohlensäure angereicherte Flüssigkeit wurde mit Queck-

1) Diese Zeitschr., Bd. XXV, S. 165. 
silberchloridlösung versetzt, bis keine Fällung mehr entstand, der Niederschlag nach mehrtägigem Stehen auf ein Filter gesammelt, mit Wasser ausgewaschen und mit Hülfe von Schwefelwasserstoff vom Quecksilber befreit, das Filtrat vom Quecksilbersulfid wurde zur Syrupconsistenz eingedampft. Die von dem Mercurichloridniederschlag abfiltrirte Flüssigkeit wurde durch Einleiten von Schwefelwasserstoff vom Quecksilber befreit, hierauf im Wasserbade etwas eingeengt und durch Zusatz von Silbernitrat von der Salzsäure befreit; auf Zusatz von Silbernitrat und Baryt entstand eine dunkle Fällung, welche das Arginin enthalten konnte. Das Filtrat vom Silberniederschlag wurde mit Salzsäure quantitativ ausgefällt; hierauf durch Schwefelsäure vom Baryt befreit und die vom Baryumsulfat getrennte Flüssigkeit mit Phosphorwolframsäure ausgefällt; diese Fällung besass die gleichen Eigenschaften, wie die zuerst erhaltene (vergl. S. 32). Diese wurde mit Baryt zersetzt und die vom Baryum befreite Lösung mit Salzsäure neutralisirt und eingedunstet.

\section{Die Identificirung der verschiedenen Basen.}

Das Histidin. Aus der bei Zerlegung des Quecksilberchloridniederschlags erhaltenen Flüssigkeit krystallisirten nach einiger Zeit harte, stark glänzende Krystalle aus, welche dem Histidinchlorid glichen. Der Chlorgehalt dieses Salzes betrug $16,03 \%$; erst nach mehrmaligem Umkrystallisiren aus Wasser wurde ein Chlorid erhalten, welches 16,80\% Chlor enthielt. Die Formel $\mathrm{C}_{6} \mathrm{H}_{9} \mathrm{~N}_{3} \mathrm{O}_{2} \mathrm{HCl}+\mathrm{H}_{2} \mathrm{O}$ verlangt $16,90 \% \mathrm{Cl}$. Die vom Chlorsilber getrennte Flüssigkeit wurde mit Silbernitrat und Ammoniak gefällt; es entstand eine amorphe weisse Fällung; der Silbergehalt derselben betrug 56,30\%. Das Histidinsilber enthält 55,77\% Ag. Das analysirte Präparat war wahrscheinlich noch nicht ganz, rein. Nach dem Auskrystallisiren des Histidinchlorids hinterblieb eine bedeutende Menge einer Mutterlauge, aus welcher bis jetzt eine krystallisirende Substanz nicht erhalten werden konnte.

Der mit Silbernitrat und Baryt erhaltene Niederschlag wurde mit Schwefelwasserstoff zersetzt und die kleinen Mengen 
von Baryt mit Schwefelsäure quantitativ ausgefällt; das Filtrat mit Salpetersäure neutralisirt und zum Syrup eingedunstet. Trotz vieler Bemühungen gelang es nicht, aus dieser Lösung Krystalle zur Abscheidung $\mathrm{zu}$ bringen; die Flüssigkeit wurde daher mit Kupferoxydhydrat gekocht, die grünblaue Lösung etwas eingedampft; auch aus dieser Kupferverbindung schieden sich keine Krystalle aus. Es wurde nun das vom Kupfer befreite Doppelsalz mit Hülfe von Silbernitrat in die Silberverbindung übergeführt; die concentrirte Lösung blieb syrupförmig; alle Versuche, ein krystallisirendes. Silbersalz zu erhalten, blieben resultatlos. Es ist aber trotzdem nicht ausgeschlossen, dass die erhaltene Basenlösung kleine Mengen Arginin einschloss, da dieselbe die für Arginin charakteristischen Fällungsreactionen gab. ${ }^{1}$ )

Die übrigen basischen Produkte. ${ }^{2}$ ) Die im Vorigen beschriebenen Substanzen machen nur einen kleinen Theil der gesammten durch Phosphorwolframsäure fällbaren Verbindungen (nach Abzug des Ammoniaks) aus, der grössere Antheil fällt auf Verbindungen, welche durch Sublimat in wässeriger Lösung bezw. Silbernitrat und Baryt nicht gefällt werden. Daher wurde nach dem Abscheiden der beiden ersten Körper eine silberreiche Flüssigkeit erhalten, welche nach dem Entfernen des gelösten Silbers und des Baryts, mit Phosphorwolframsäure einen recht starken Niederschlag gab; derselbe besass die gleiche Beschaffenheit wie der Anfangs erhaltene. Aus diesem wurde in bekannter Weise eine sehr stark alkalisch reagirende Flüssigkeit erhalten, welche nach dem Neutralisiren mit Salzsäure und Eindampfen zum Syrup nach einigen Stunden krystallinisch erstarrte. Die Quantität dieser Krystalle betrug $15 \mathrm{~g}$ aus $6^{1 / 2} \mathrm{~kg}$ geblähtem Käse. Aus dem wässerigen Extract des entfetteten Käses wurden noch weitere $2 \mathrm{~g}$ einer solchen Substanz erhalten.

1) Möglicher Weise schliesst die mit $\mathrm{AgNO}_{3}$ und $\mathrm{Ba}(\mathrm{OH})_{2}$ erhaltene Fällung Guanidin ein, da dasselbe, wie beobachtet, unter Umständen von Silbernitrat bei Gegenwart von Basen gefällt wird.

2) Da Herr Thöni die Arbeit abbrechen musste, wurde dieser Theil der vorliegenden Arbeit vom Berichterstatter allein ausgeführt. 
Die wässerige Lösung dieser Krystalle ist rechtsdrehend: $0,417 \mathrm{~g}$ in $10 \mathrm{ccm}$. gelöst drehten im $20 \mathrm{~mm}$.-Rohr im SoleilVentzke'schen Apparat $+2^{0} .13 \mathrm{~g}$ dieser Substanz ${ }^{1}$ ) wurden mit einem Gemisch von Alkohol und Aether behandelt, bis nichts mehr in Lösung ging; diese Lösung wurde in der unten zu beschreibenden Weise weiter verarbeitet. Es hinterblieben nun $10 \mathrm{~g}$ einer fast farblosen, anscheinend einheitlichen Krystallmasse, dieselbe wurde in wenig Wasser gelöst, von den dabei verbleibenden minimalen Mengen eines amorphen Rückstandes getrennt und die Lösung mit circa $2 \mathrm{~g}$ einer alkoholischen Platinchloridlösung versetzt; es schieden sich sofort Krystalle aus, welche nach 24 stündigem Stehen von der Flüssigkeit getrennt und aus Alkohol umkrystallisirt wurden. Diese Krystalle waren nicht einheitlicher Natur, neben sechsseitigen büschelförmig angeordneten langgestreckten Blättchen traten grössere anscheinend vierseitige, deutlich ausgebildete Prismen auf, dazwischen waren die charakteristischen Oktaeder des Kaliumplatinchlorids eingestreut. Durch wiederholtes Umkrystallisiren aus Wasser bezw. verdünntem Alkohol wurden sodann Krystalle erhalten, welche sich beim Betrachten unter dem Mikroskop als nahezu einheitlich erwiesen. Der Platingehalt derselben betrug $39,27 \%$.

Aus der Mutterlauge von dem zuerst ausgefällten Platindoppelsalz wurden durch partielles Fällen mit alkoholischer Platinchloridlösung noch weitere 4 Fractionen von Doppelsalzen erhalten. Die Gesammtausbeute betrug 4 g. Der Platingehalt der einzelnen Krystallfractionen betrug nach einmaligem Umkrystallisiren $39,37 \%, 39,11 \%, 38,95 \%$ und $38,49 \%$. Die bei der Zerlegung der Platindoppelsalze erhaltenen Chlorhydrate wurden gesondert zum Syrup eingedunstet; die Verdampfungsrückstände wurden einige Male mit kleinen Quantitäten 95\% igen Alkohols behandelt; die alkoholischen Auszüge wurden alle vereinigt und in Exsiccator zur Verdunstung aufgestellt; es hinterblieb eine zum Theil krystallinische Masse, welche sich in 95\% igem Alkohol bis auf einen minimalen Rückstand auflöste; diese Lösung

1) $4 \mathrm{~g}$ davon waren für Vorversuche verbraucht. 
wurde mit einer concentrirten alkoholischen Platinchloridlösung versetzt; die ausgeschiedenen Krystalle wurden einmal umkrystallisirt. Der Stickstoffgehalt derselben betrug 5,55\%, der Platingehalt $38,33 \%$.

$0,0955 \mathrm{~g}$ bei $105^{\circ}$ getrockneter Krystalle gaben $4,8 \mathrm{ccm}$. Gas bei $16^{\circ}$ und $720 \mathrm{~mm}$. Druck $=0,0053 \mathrm{~g} \mathrm{~N}$.

$0,2100 \mathrm{~g}$ bei $105^{\circ}$ getrockneter Krystalle gaben $0,0805 \mathrm{~g} \mathrm{Pt}$.

Der Stickstoff- und Platingehalt stimmen somit auf das Chlorplatinat des Pentamethylendiamins. Die vom Platin befreite Verbindung zeigte alle die Fällungsreactionen, welche dem Pentamethylendiamin zukommen.

Das bei obiger Extraction mit $95 \%$ igem Alkohol verbliebene nicht hygroskopische Chlorhydrat wurde ebenfalls in das Platindoppelsalz übergeführt; derselbe erwies sich als schwer löslich im Alkohol und nicht leicht löslich im Wasser. Die einmal umkrystallisirte Verbindung bestand aus wohl ausgebildeten sechsseitigen Täfelchen, ${ }^{1}$ ) sie enthielt $5,62 \%$ Stickstoff und 39,44\% Platin. Die Formel $\mathrm{C}_{4} \mathrm{H}_{14} \mathrm{~N}_{2} \mathrm{PtCl}_{6}$ verlangt $39,14 \% \mathrm{Pt}$ und $5,63 \% \mathrm{~N}$.

$0,1168 \mathrm{~g}$ bei $105^{\circ}$ getrockneter Krystalle gaben $5,8 \mathrm{ccm}$. $\mathrm{N}$ bei $16^{n}$ und $738 \mathrm{~mm}$. Druck $=0,00656 \mathrm{~g} \mathrm{~N}$.

$0,451 \mathrm{~g}$ bei $105^{\circ}$ getrockneter Krystalle gaben $0,1779 \mathrm{~g} \mathrm{Pt}$.

Die vom Platinsulfid getrennte Flüssigkeit wurde nach dem Vertreiben des Schwefelwasserstoffs für eine Chlorbestimmung benutzt; es wurden $41,3 \%$ Chlor gefunden.

Der Stickstoff- und Platingehalt dieses in sechsseitigen Blättchen krystallisirenden Chlorplatinats stimmen gut auf das Platindoppelsalz des Putrescins (Tetramethylendiamins). Der Chlorgehalt wurde etwas zu niedrig gefunden, wahrscheinlich weil kleine Mengen von Chlorwasserstoff beim Entfernen des $\mathrm{H}_{2} \mathrm{~S}$ mit ausgetrieben wurden. Das aus dem Platinsalz erhaltene Chlorhydrat gab folgende Reactionen:

Phosphorwolframsäure, Phosphormolybdänsäure, krystallinische Fällungen, Kaliumwismuthjodid, rothe krystallinische

1) Ueber die Krystallform des Tetramethylendiamins vergleiche man G. Ciamician und C. U. Zanetti, Ber. d. deutsch. chem. Gesellsch., Ḅd. 22, S. 1968. 
Fällung. Goldchlorid gelben krystallinischen Niederschlag. Charakteristisch für Tetramethylendiaminchlorhydrat ist das Auftreten einer anfänglich öligen, dann rasch krystallinisch erstarrenden blätterigen Masse auf Zusatz von Kaliumquecksilberjodid: Mit Nessler'schem Reagens trat eine amorphe, schwach gelbe Fällung auf. Pikrinsäure erzeugt ein in Nadeln anschiessendes Salz, welches, aus Wasser umkrystallisirt, lange, stark glänzende, schwach dichroitische Nadeln bildet.

Das Lysin. Die von den schwer löslichen Platinsalzen getrennte Flüssigkeit wurde stark concentrirt, sodann mit viel absolutem Alkohol versetzt, bis eine Trübung auftrat; der Platingehalt der dabei ausgeschiedenen Krystalle, welche im Aussehen mit Lysinplatinchlorid übereinstimmten, betrug $34,95 \%$.

$0,1614 \mathrm{~g}$ bei $120^{\circ}$ getrockneter Krystalle gaben $0,0564 \mathrm{~g}$ Pt.

Das Lyssin ist von Steinegger ${ }^{1}$ ) aus den sogenannten Salzsteinen des Emmenthaler Käses isolirt worden.

Das Guanidin. Das durch Behandeln der Cihlorhydrate mit Alkoholäther erhaltene Extract wurde verdunstet, der Verdampfungsrückstand in Wasser gelöst und mit einer Goldchloridlösung fractionirt ausgefällt, von der zuerst auftretenden amorphen Fällung wurde abfiltrirt, die auf weiteren Zusatz von Goldlösung entstandenen feinen Nadeln wurden einmal aus Wasser umkrystallisirt. Der Goldgehalt derselben betrug 49,79\%. Die Formel $\mathrm{CH}_{5} \mathrm{~N}_{3} \cdot \mathrm{HCl} \cdot \mathrm{AuCl}_{3}$ verlangt 49,45 \% $\mathrm{Au}$.

$0,1400 \mathrm{~g}$ exsiccatortrockenes Golddoppelsalz gaben 0,0697 $\mathrm{g} \mathrm{Au}$.

Die durch Zerlegen des Goldsalzes mit Schwefelwasserstoff erhaltene saure Lösung wurde durch Einleiten von Luft vom Schwefelwasserstoff befreit, mit Phosphorwolframsäure gefällt; die durch Zersetzen mit Baryt erhaltene basische Flüssigkeit wurde vom Baryum befreit, mit Pikrinsäure neutralisirt und das ausgeschiedene Pikrat einmal aus Wasser umkrystallisirt, dasselbe zersetzte sich nahezu gleichzeitig wie ein aus Guanidinchlorid dargestelltes Präparat bei $316-318^{\circ}$.

1) R. S t e in e g g er, Die Salzsteine, ihre chemische Zusammensetzung, Bildung und Vermeidung. Landw. Jahrbuch der Schweiz, Bd. 15, S. 132. 
Unter den Spaltungsprodukten des Paracaseins im Emmenthaler Käse lassen sich also neben Aminosäuren basische Produkte, nämlich Ammoniak, Histidin, Lysin, Pentamethylendiamin und Tetramethylendiamin, nachweisen, ausserdem ist das Vorhandensein von Guanidin sehr wahrscheinlich. F. Benecke und E. Schulze1) fanden, dass beim Erhitzen des entfetteten Käses mit Magnesiamilch bei wiederholter Destillation ein immer wieder alkalisch reagirendes Destillat übergeht. Eine Beobachtung, die wir bestätigen können. Dieser Befund findet durch den Nachweis flüchtiger Basen im Käse vielleicht eine Erklärung. Es soll daher geprüft werden, das Tetramethylendiamin und das Pentamethylendiamin durch Destillation mit Magnesia von den anderen nicht flüchtigen Basen zu trennen; um das dabei gleichzeitig übergehende Ammoniak wegzuschaffen, kann man die von Brieger angegebene Fällung der Chloride mit Sublimatlösung benutzen. Arginin konnte nicht isolirt werden; man wird daher schliessen dürfen, dass das bei der Spaltung des Paracaseins primär gebildete Arginin einer weiteren Zersetzung anheim gefallen ist und dass dabei Guanidin und Tetramethylendiamin entstanden sind. Es ist ja bekannt, dass das bei der Spaltung des Arginins entstehende Ornithin unter Mitwirkung von Fäulnissorganismen Putrescin liefert. ${ }^{2}$ ) Kutscher ${ }^{3}$ ) fand, dass bei der Oxydation des Arginins Guanidin gebildet wird. Als die Muttersubstanz des Cadaverins darf das Lysin angesehen werden. Das letztere geht bei der Fäulniss unter Abspaltung von Kohlensäure in Pentamethylendiamin über. ${ }^{4}$ )

Der Berichterstatter (E. W.) hat noch eine Reihe verschiedener Emmenthaler Käsesorten in angegebener Weise verarbeitet. In allen den untersuchten Materialien konnte eine in Alkohol lösliche, durch Wasser fällbare Eiweisssubstanz (Caseoglutin), daneben auch Peptone nachgewiesen werden.

1) Untersuchungen über den Emmenthaler Käse und über einige andere schweizerische Käsesorten. Landw. Jahrbücher 1887, S. 344.

2) und 4) A. Ellinger, Zur Constitution des Ornithins und des Lysins. Zugleich ein Beitrag zur Chemie der Eiweissfäulniss. Diese Zeitschrift, Bd. XXIX, S. 334.

3) Fr. Kutscher, Die Oxydationsprodukte des Arginins. Diese Zeitschrift, Bd. XXXII, S. 413. 
Aminosäuren wurden stets in beträchtlicher Menge vorgefunden; beachtenswerth ist jedoch, dass das Tyrosin stets nur in kleinen Mengen auftrat. Nur in einem Falle - aus einem sehr alten Käse - wurde diese Aminosäure in grösserer Menge isolirt, dieselbe erwies sich jedoch viel schwächer rechtsdrehend $\left(\alpha_{\mathfrak{D}}=+6,4^{\circ}\right)$, als das durch Zersetzen von Eiweiss durch Säuren erhaltene Tyrosin. Unter den basischen Produkten konnten stets Ammoniak und Histidin nachgewiesen werden; in einem Falle wurden beträchtliche Mengen Lysin als Platindoppelsalz erhalten. Das Arginin wird beim Reifen des Käses anscheinend rasch verbraucht. Es wurde bisher aus keiner der untersuchten Käsesorten in reinem Zustande erhalten. Dieser Befund stimmt in mancher Beziehung mit den Ergebnissen überein, welche D. Lawrow ${ }^{1}$ ) bei andauernder peptischer Verdauung von Eiweissstoffen erhalten hat.

Die Vertheilung des Stickstoffs auf die verschiedenen Spaltungsprodukte des Paracaseins ist also in den verschiedenen Käsesorten eine verschiedene.

Es ist beabsichtigt, diese Arbeit durch die Trennung und Untersuchung der einzelnen Aminosäuren zu vervollständigen. Es wird ferner zu prüfen sein, ob neben den erwähnten Basen sich auch noch andere basische Eiweisszerfallsprodukte, Ornithin, Phenyläthylamin und Oxyphenyläthylamin, nachweisen lassen. Auch dürfte es angezeigt sein, die von E. Schulze begonnene Untersuchung über das Caseoglutin fortzusetzen; vielleicht gelingt es, durch eine solche Untersuchung in gewisser Hinsicht Aufschluss über das Casein zu erhalten. Eine ausführliche Mittheilung über die Bestandtheile des Emmenthaler Käses soll in dem landwirthschaftlichen Jahrbuch der Schweiz erfolgen.

1) D. Lawrow, Zur Kenntniss des Chemismus der peptischen und tryptischen Verdauung der Eiweisskörper. Diese Zeitschr., Bd. XXXIII, S. 312.

Man vergleiche über diesen Gegenstand ferner die Arbeiten von L. Emerson, Ueber das Auftreten von Oxyphenyläthylamin bei Pankreasverdauung und über fermentative $\mathrm{CO}_{\mathbf{z}}$-Abspaltung, Hofmeister's Beiträge, Bd. 1 , S. 501 .

L. Langstein, Zur Kenntniss der Endprodukte der peptischen Verdauung, ibid. Bd. 1, S. 507. 